\title{
Glossary to Support Applied International Research on Decision Making for High Conflict Urban Marches and Parades
}

\author{
Liane Hauff ${ }^{1}$. Thibaut Heckmann ${ }^{2}$ \\ ${ }^{1}$ Hochschule der Polizei Rheinland-Pfalz, Büchenbeuren, Germany \\ E-mail: liane_hauff@hotmail.com \\ ${ }^{2}$ Centre de Recherche de l'Ecole des Officiers de la Gendarmerie Nationale, Melun, France \\ E-mail: thibaut.heckmann@gendarmerie.interieur.gouv.fr
}

Received: 5 May 2021 / Accepted: 10 July 2021

DOI: $10.17815 / \mathrm{CD} .2021 .113$

\begin{abstract}
This article was created in context of OPMoPS (Organized Pedestrian Movement in Public Spaces), a French-German interdisciplinary collaboration on high conflict urban marches and parades. As OPMoPS aims to support decision making for authorities of public order, both a French and a German police institution are members of the consortium. Communication with target group was insofar challenging, as their experts' language is close to everyday terms. Thus the authors are proposing the following glossary to support applied international research in this field. Both authors are not skilled language experts but pragmatic members of OPMoPS's police institutions.

All terms can be found in English, German and French, with a focus on police and on German police procedure. It is firstly classed in thematic order, and secondly in alphabetical order.
\end{abstract}

Keywords Glossary · demonstration · parade $\cdot$ police $\cdot$ tactic $\cdot$ procedure $\cdot$ march . authorities of public order

\section{Introduction}

Marches and parades are a regular phenomenon in urban spaces. At such events, citizens make use of their basics rights to express public matters or to enjoy themselves. It is a well-known fact, that marches and parades raise conflicts and questions of security: Very often demonstrators and counter-demonstrators face each other or crowds of visitors become more and more dense at entry points. The German Federal Ministry of Education 
and Research (BMBF) and the French National Agency of Research (ANR) started a program to fund research projects to address questions of future safety in urban spaces. With "Organized Pedestrian Movement in Public Spaces" (OPMoPS, September 2017 to May 2021), both organisations support an interdisciplinary and French-German consortium researching marches and parades. Experts from computer science, mathematics, sociology and the law are working together to make such events safer.

OPMoPS is a project of applied science. The goal of OPMoPS is to support members of authorities of public order with their decision making when preparing for marches and parades. Authorities of public order are represented in OPMoPS by the University of Police Rhineland-Palatinate, Germany, and the Research Center of the National Gendarmerie's University, France. Results from OPMoPS are being integrated in a demonstrator of a digital platform which works as a decision support system (DSS), mainly by visualizing alternatives and their consequences.

Growing together as one team at OPMoPS, all had to learn about the methods, tools and vocabulary of the different represented disciplines. Also, the consortium made a point to exchange with potential users of the DSS. Soon it became obvious, that it is important to use correct terminology - the terminology of members of police and other authorities of public order, which is necessarily quite different from the terminology of other OPMoPS members. Also, this terminology seems to be close to everyday terms, but has, in fact, very different meanings. There are a number of specific dictionaries which contain, for example, police terminology. However, they emphasize on ranks and organizations, not on procedures. Procedure is what answers the question of How can be assured that citizens can make use of their basic rights, but safely? This is exactly the question which needs to be answered when preparing for marches and parades. We needed to be able to discuss our results. Thus the idea of this glossary was born.

The authors are representatives of the police institutions at OPMoPS and created the glossary by use of dictionaries and encyclopedia [1-5], specialized dictionaries [6-10], and police resources, which are not available for the public. All terms can be found in English, German and French, with a focus on police and on German police procedure. It is firstly classed in thematic order, and secondly in alphabetical order. 


\section{Authorities and Jurisdiction}

1 (to) announce a demonstration or an event - (to) inform authorities you're planning a demonstration or an event
- eine Versammlung oder Veranstaltung bei der zuständigen Behörde anmelden

I déclaration préalable - informer les autorités et déclarer une manifestation ou événement public à la préfecture
2 authorities of public order emergency services (in a broad sense)
Behörden mit Sicherheits- und Ordnungsaufgaben (BOS) - Behörden, die für die öffentliche Sicherheit und Ordnung zuständig sind

I autorité publique - service d'urgence (au sens large)
3 basic rights

4 by-law - a rule adopted by a local authority
Grundrechte

droits fondamentaux

Verordnung - von einer Behörde erlassene Vorschrift

I arrêté - loi appliquée par les autorités locales
5 constitution (for Germany: basic law)

Verfassung (für Deutschland: Grundgesetz)

II constitution

6 cooperation meeting between organizers, local authority and police, to make a demonstration or a public event possible despite risks
Kooperationsgespräch - Gespräch zwischen Verantwortlichen, um eine Versammlung, auch trotz konkreter Risiken, zu ermöglichen

1 réunion d'information - entre les organisateurs de la manifestation, les autorités locales et la prefecture pour préparer la manifestation/l'événement public en prévoyant les risques à 1'ordre public

7 law of assembly - law specifying freedom of demonstration
Versammlungsgesetz

I droit de rassemblement - loi spécifiant la liberté de manifestation 
8 local authority - municipal institution
Behörde; hier: Versammlungsbehörde

I autorités locales - autorités terrotorialement compétentes
9 (to) maintain public order die öffentliche Sicherheit und Ordnung sicherstellen

I le maintien de l'ordre public

10 marshal - a private person appointed by the organizer of an event to guarantee safety

Ordner - vom Versammlungsleiter, der weitgehend selbst für die Einhaltung von Auflagen verantwortlich ist, zur Unterstützung bestimmte Person

I vigiles - l'organisateur est en grande partie responsable du respect des exigences de sécurité et peut nommer des vigiles à cette fin

Polizei- und Ordnungsbehördengesetz (für das Land Rheinland-Pfalz)

I le cadre légal - loi régissant les forces de l'ordre

Auflagen - Vorgaben (für den Versammlungsleiter bzw. Organisator) für die Durchführung einer Versammlung oder Veranstaltung, sodass diese trotz bekannter Risiken möglich ist

I restrictions - moyens juridiques pour réduire le risque spécifique lié au rassemblement de personnes, l'organisateur est garant de leur respect

\section{Demonstrations and Parades}

13 black bloc - demonstrators in black clothing with concealed faces, often acting to overthrow public order
- Schwarzer Block - schwarz gekleidete und vermummte, oft gewaltbereite Aktivisten

I black bloc - fauteur de trouble vêtu de noir et/ou visage masqué 
14 (to) block (street)

blockieren (Straße)

1 bloquer (rue)

\begin{tabular}{|c|c|c|}
\hline 15 & blockade & $\begin{array}{l}\text { Blockade } \\
\text { blocus }\end{array}$ \\
\hline 16 & bottleneck & $\begin{array}{l}\text { Engstelle } \\
\text { goulot d'étranglement }\end{array}$ \\
\hline 17 & counter-demonstration & $\begin{array}{l}\text { Gegen-Demonstration } \\
\text { contre-manifestation }\end{array}$ \\
\hline 18 & criminal damage & $\begin{array}{l}\text { Sachbeschädigung } \\
\text { dégradation }\end{array}$ \\
\hline 19 & crowd & $\begin{array}{l}\text { Menschenmenge } \\
\text { foule }\end{array}$ \\
\hline 20 & crowded & $\begin{array}{l}\text { gedrängt } \\
\text { bondé (saturé) }\end{array}$ \\
\hline 21 & demonstrator & $\begin{array}{l}\text { Demonstrant } \\
\text { manifestant }\end{array}$ \\
\hline 22 & $\begin{array}{l}\text { demonstration, rally - a public } \\
\text { display of group feelings } \\
\text { toward a person or cause }\end{array}$ & $\begin{array}{l}\text { Versammlung } \\
\text { manifestation }\end{array}$ \\
\hline 23 & $\begin{array}{l}\text { (to) deviate from the planned } \\
\text { route }\end{array}$ & $\begin{array}{l}\text { von der geplanten Strecke abweichen } \\
\text { dévier de la trajectoire planifée }\end{array}$ \\
\hline 24 & (to) disrupt & $\begin{array}{l}\text { stören } \\
\text { perturber }\end{array}$ \\
\hline 25 & $\begin{array}{l}\text { disruption (traffic, } \\
\text { demonstration) }\end{array}$ & $\begin{array}{l}\text { Störung (Verkehr, Versammlung) } \\
\text { perturbation (trafic, démonstration) }\end{array}$ \\
\hline 26 & $\begin{array}{l}\text { left(-wing) - extremist left } \\
\text { political position }\end{array}$ & $\begin{array}{l}\text { linksextrem } \\
\text { extrémistes de gauche }\end{array}$ \\
\hline 27 & (to) loot & $\begin{array}{l}\text { plündern } \\
\text { piller }\end{array}$ \\
\hline
\end{tabular}




\begin{tabular}{|c|c|c|}
\hline 28 & $\begin{array}{l}\text { march - a form of } \\
\text { demonstration, organized } \\
\text { procession of demonstrators }\end{array}$ & $\begin{array}{l}\text { Aufzug } \\
\text { défilé }\end{array}$ \\
\hline 29 & moderate-left & $\begin{array}{l}\text { bürgerlich-links } \\
\text { gauche modérée }\end{array}$ \\
\hline 30 & moderate-right & $\begin{array}{l}\text { bürgerlich-rechts } \\
\text { droite modérée }\end{array}$ \\
\hline 31 & $\begin{array}{l}\text { parade - a form of public } \\
\text { event, a group of individuals } \\
\text { moving along in an orderly } \\
\text { often ceremonial way }\end{array}$ & $\begin{array}{l}\text { Umzug, Parade - ein festlicher, ge- } \\
\text { meinsamer Marsch } \\
\text { parade - défilé festif (carnaval) }\end{array}$ \\
\hline 32 & participant & $\begin{array}{l}\text { Teilnehmer } \\
\text { participant }\end{array}$ \\
\hline 33 & $\begin{array}{l}\text { paving stone (may be used as } \\
\text { projectiles against police) }\end{array}$ & $\begin{array}{l}\text { Pflasterstein (als Wurfmaterial) } \\
\text { pavé (peut être utilisé comme arme } \\
\text { contre les forces de l'ordre) }\end{array}$ \\
\hline 34 & peaceful & $\begin{array}{l}\text { friedlich } \\
\text { pacifique }\end{array}$ \\
\hline 35 & $\begin{array}{l}\text { pedestrian stream - a } \\
\text { multitude of pedestrians } \\
\text { walking generally in the same } \\
\text { direction }\end{array}$ & $\begin{array}{l}\text { Fußgängerstrom } \\
\text { flux de pietons }\end{array}$ \\
\hline 36 & $\begin{array}{l}\text { procession of demonstrators - } \\
\text { a group of protestors moving } \\
\text { mainly in one direction }\end{array}$ & $\begin{array}{l}\text { Demonstrationszug } \\
\text { cortège de manifestants }\end{array}$ \\
\hline 37 & protest & $\begin{array}{l}\text { Protest } \\
\text { contestation }\end{array}$ \\
\hline 38 & (to) protest & $\begin{array}{l}\text { protestieren } \\
\text { contester }\end{array}$ \\
\hline 39 & (to) provoke & $\begin{array}{l}\text { provozieren } \\
\text { provoquer }\end{array}$ \\
\hline 40 & $\begin{array}{l}\text { public event - an (outdoor) } \\
\text { entertainment }\end{array}$ & $\begin{array}{l}\text { Veranstaltung } \\
\text { événement public }\end{array}$ \\
\hline
\end{tabular}


41 public transport - tramway, omnibus, underground, train
Öffentlicher Personen-Nahverkehr (ÖPNV) - Straßenbahn, Bus, U-Bahn, Bahn

Iransport public - bus, métro, train

\begin{tabular}{|c|c|c|}
\hline 42 & $\begin{array}{l}\text { rally, demonstration - a public } \\
\text { display of group feelings } \\
\text { toward a person or cause }\end{array}$ & $\begin{array}{l}\text { Versammlung } \\
\text { manifestation }\end{array}$ \\
\hline 43 & $\begin{array}{l}\text { right(-wing) - extremist right } \\
\text { political position }\end{array}$ & $\begin{array}{l}\text { rechtsextrem } \\
\text { extrémistes de droite }\end{array}$ \\
\hline 44 & route (march, parade) & $\begin{array}{l}\text { (Verlaufs-)Strecke } \\
\text { itinéraire (marche, défilé) }\end{array}$ \\
\hline 45 & routing & $\begin{array}{l}\text { Festlegung einer Strecke } \\
\text { routage }\end{array}$ \\
\hline 46 & slogan & $\begin{array}{l}\text { Motto } \\
\text { slogan }\end{array}$ \\
\hline 47 & speech & $\begin{array}{l}\text { Kundgebung } \\
\text { discours de revendications }\end{array}$ \\
\hline 48 & $\begin{array}{l}\text { street furniture - objects } \\
\text { installed in public places, } \\
\text { example: benches }\end{array}$ & $\begin{array}{l}\text { Straßenmöbel - möbelähnliche Ge- } \\
\text { genstände im öffentlichen Raum, Bei- } \\
\text { spiel: Bänke } \\
\text { mobilier urbain - objets installés dans } \\
\text { l'espace public }\end{array}$ \\
\hline 49 & $\begin{array}{l}\text { trouble maker - demonstrator } \\
\text { who acts willingly against the } \\
\text { law }\end{array}$ & $\begin{array}{l}\text { Störer - Demonstrationsteilnehmer, } \\
\text { dessen Absicht es bekanntermaßen ist, } \\
\text { gegen Gesetze zu verstoßen } \\
\text { fauteur de trouble - personne qui } \\
\text { trouble 1'ordre public }\end{array}$ \\
\hline 50 & $\begin{array}{l}\text { urban guerilla - unofficial } \\
\text { group fighting government } \\
\text { with unconventional or violent } \\
\text { means in urban environment }\end{array}$ & $\begin{array}{l}\text { Urban Guerilla (kein Phänomen in } \\
\text { Deutschland) } \\
\text { guérilla urbaine - les combattants se } \\
\text { battent contre un gouvernement en mi- } \\
\text { lieu urbain }\end{array}$ \\
\hline
\end{tabular}


51 venue - where a public event or demonstration takes place
Veranstaltungsort

I lieu principal de la manifestation - là où a lieu un événement public

\section{Authorities of Public Order at Work}

52 briefing - a meeting to prepare personnel for the forthcoming operation
- Einsatzbesprechung - Einweisung in die bevorstehende Lage und Aufträge

I réunion pré-opératoire - une réunion pour préparer le personnel pour la mission à venir
53 commanding officer - a senior officer, responsible to overcome the current situation according to the operation plan he or she approved
Einsatzleiter - ist für den laufenden Einsatz verantwortlich

I commandant des opérations - officier responsable du déroulement des opérations, donne son approbation et ses directives
54 debriefing - a meeting to evaluate the past operation
Einsatznachbereitung - eine Besprechung, bei der der vergangene Einsatz reflektiert wird

I analyse de bonnes pratiques - une réunion pour évaluer la mission et en tirer un enseignement pour les futures missions

55 decision support system Entscheidungsunterstützungssystem

II système d'aide à la décision

56 fire fighter

- Feuerwehrmann/-frau

I sapeur pompier

57 first responder - a person who is among those responsible for going immediately to the scene of an accident or emergency to provide assistance
! First Responder ist in Deutschland mit „medizinischen Ersthelfern“ (qualifizierte Laien) belegt !

1 premier intervenant/primo arrivant une personne qui est capable de se rendre immédiatement sur les lieux d'un incident ou d'une urgence pour porter assistance 
58 operators - all personnel from police deployed to cope with a situation (general expression: personnel)

\begin{tabular}{|c|c|c|}
\hline 59 & paramedic & $\begin{array}{l}\text { Rettungshelfer } \\
\text { Unités Médicales de Secours }\end{array}$ \\
\hline 60 & personnel & $\begin{array}{l}\text { Einsatzkräfte } \\
\text { membres opérationnels - l'ensemble } \\
\text { du personnel déployé pour faire face à } \\
\text { une situation }\end{array}$ \\
\hline 61 & (police) officer & $\begin{array}{l}\text { Polizeibeamte } / \mathrm{r} \\
\text { officier de police }\end{array}$ \\
\hline 62 & $\begin{array}{l}\text { tactical equipment }- \text { sum of } \\
\text { means that can be used to } \\
\text { overcome a situation } \\
\text { (example: radio) }\end{array}$ & $\begin{array}{l}\text { Einsatzmittel - technische und physi- } \\
\text { sche Hilfsmittel (Beispiel: Funk) } \\
\text { équipement tactique - moyen maté- } \\
\text { riel pour appuyer les forces de l'ordre } \\
\text { (exemple: radio) }\end{array}$ \\
\hline
\end{tabular}

\section{Einsatzkräfte}

I membres opérationnels

\subsection{Procedure}

63 access point

64 access road 
69 (to) assign to (task, personnel) einteilen (von Einsatzmitteln und -kräften gemäß einem Auftrag)

I mission à accomplir (tache, personnel)

70 (to) be in charge zuständig sein

I être en charge de

71 BFE - in Germany a task unit specialised in making arrests from within groups of civilians
BFE (Beweis- und Festnahmeeinheit) - für Zugriffe in Menschenmengen ausgebildete Einheit

I BFE - unité allemande formée pour les opérations de contrôle des foules

72 calling distance

- Rufweite (innerhalb/außerhalb)

(within/beyond) - guideline

1 dans/en dehors du champs d'action opposing groups

73 (to) carry - (to) keep operators or tactical equipment close to a moving situation, opposite to: stationary mitführen von Einsatzkräften oder Einsatzmitteln

I mobiliser - pour garder des forces opérationnelles à proximité d'une situation tendue
74 cordon - police procedure to close public space (example: street) for passing persons, things or vehicles
Absperrung - Taktik, um das Passieren von Personen, Fahrzeugen, Dingen zu verhindern

I cordon de sécurité - procédure de maintien de l'ordre pour fermer un espace au public (exemple: rue)

75 (to) cordon off

eine Straße absperren

- bouclage (rue)

76 (to) deploy $^{1}$ (to a location, of personnel or technical equipment)

einsetzen (von Einsatzkräften oder -mitteln)

I déployer (en lieu, personnel ou équipements)

77 (to) deploy ${ }^{2}$ verlagern (von Einsatzkräften oder -mitteln)

re-déploiement 
78 (to) detain - to arrest someone preliminary festnehmen (vorläufig, Beispiel: zur Identitätsfestellung)

IInterpeller - emmener une personne suspectée de crime ou délit dans une unité de police ou gendarmerie
79 (to) deter someone from doing something jemand von etwas abhalten, etwas verhindern

I dissuader quelqu'un de faire quelque chose

\begin{tabular}{|c|c|c|}
\hline 80 & $\begin{array}{l}\text { detour - a temporary } \\
\text { alternative route }\end{array}$ & $\begin{array}{l}\text { (Verkehrs-)Umleitung } \\
\text { déviation }\end{array}$ \\
\hline 81 & (to) disperse a demonstration & $\begin{array}{l}\text { eine Versammlung auflösen } \\
\text { disperser une manifestation }\end{array}$ \\
\hline 82 & $\begin{array}{l}\text { duty roster - tool to deploy } \\
\text { personnel to specific tasks, is } \\
\text { part of general orders }\end{array}$ & $\begin{array}{l}\text { Diensteinteilung } \\
\text { cahier des charges }\end{array}$ \\
\hline 83 & $\begin{array}{l}\text { emergency response (usually } \\
\text { not police) }\end{array}$ & $\begin{array}{l}\text { schnelles Eingreifen } \\
\text { II réponse urgente }\end{array}$ \\
\hline 84 & $\begin{array}{l}\text { emergency vehicle - cars with } \\
\text { specific features }\end{array}$ & $\begin{array}{l}\text { Einsatzfahrzeug } \\
\text { véhicule d'urgence }\end{array}$ \\
\hline 85 & $\begin{array}{l}\text { estimate of the situation - } \\
\text { analysis of a situation }\end{array}$ & $\begin{array}{l}\text { Beurteilung der Lage } \\
\text { analyse de la situation }\end{array}$ \\
\hline 86 & $\begin{array}{l}\text { general order }(s)-\text { sum of } \\
\text { orders to overcome a specific } \\
\text { situation }\end{array}$ & $\begin{array}{l}\text { Einsatzbefehl } \\
\text { ordres préparatoires }\end{array}$ \\
\hline 87 & guidelines & $\begin{array}{l}\text { Leitlinien } \\
\text { guides des bonnes pratiques }\end{array}$ \\
\hline 88 & $\begin{array}{l}\text { head of task force }- \text { a task } \\
\text { force's designated leader }\end{array}$ & $\begin{array}{l}\text { Abschnittsleiter } \\
\text { chef d'opération }\end{array}$ \\
\hline
\end{tabular}


89 immediate situation - the kind of situation which calls for immediate action while leaving nearly no time to estimate and/or prepare the operation, opposite to: notice situation
Sofortlage - eine Lage, bei der wegen der kurzen Vorlaufzeit zeitnahes und teilweise improvisiertes Eingreifen erforderlich ist

I ordre en cours d'action - situation qui necessite une action immédiate ne laissant pas de place à l'anticipation (opposé à l'ordre initial)

\begin{tabular}{|c|c|c|}
\hline 90 & intersection & $\begin{array}{l}\text { Kreuzung } \\
\text { carrefour }\end{array}$ \\
\hline 91 & $\begin{array}{l}\text { (to) intervene (especially } \\
\text { police) }\end{array}$ & $\begin{array}{l}\text { eingreifen (der Polizei) } \\
\text { intervenir (généralement la police) }\end{array}$ \\
\hline 92 & location & $\begin{array}{l}\text { (aktueller) Standort } \\
\text { emplacement }\end{array}$ \\
\hline 93 & (to) monitor (example: traffic) & $\begin{array}{l}\text { überwachen } \\
\text { surveiller }\end{array}$ \\
\hline 94 & $\begin{array}{l}\text { notice situation - the kind of } \\
\text { situation which leaves } \\
\text { authorities of public order time } \\
\text { to prepare the operation, due to } \\
\text { an early announcement, } \\
\text { opposite to: immediate } \\
\text { situation }\end{array}$ & $\begin{array}{l}\text { Zeitlage - eine Lage, die frühzeitig } \\
\text { absehbar ist, so dass umfangreiche } \\
\text { Einsatzvorbereitungen und notwendi- } \\
\text { ge Abstimmungen erfolgen können } \\
\text { ordre initial - rédigé en avance de } \\
\text { l'évenement annoncé, il permet au } \\
\text { commandant des opérations de diffu- } \\
\text { ser ses ordres (opposé à l'ordre en } \\
\text { cours d'action) }\end{array}$ \\
\hline 95 & on scene & $\begin{array}{l}\text { vor Ort } \\
\text { sur place }\end{array}$ \\
\hline 96 & $\begin{array}{l}\text { operation - a usually military } \\
\text { action, mission, or maneuver } \\
\text { including its planning and } \\
\text { execution }\end{array}$ & $\begin{array}{l}\text { Einsatz } \\
\text { opération }\end{array}$ \\
\hline 97 & $\begin{array}{l}\text { order - a specific mission with } \\
\text { which a person or a group is } \\
\text { charged, also specifies how } \\
\text { mission is to be accomplished, } \\
\text { see also: task }\end{array}$ & $\begin{array}{l}\text { Befehl } \\
\text { ordre }\end{array}$ \\
\hline
\end{tabular}


98 (to) overcome a situation - (to) end a (critical) situation by gaining and maintaining control

\begin{tabular}{lll}
\hline $99 \begin{array}{l}\text { (to) patrol - being present on a } \\
\text { destined area to detect and } \\
\text { assure security }\end{array}$ & bestreifen \\
patrouiller
\end{tabular}


107 route security - police procedure to make sure a route is free for the intended purpose
- Streckenschutz - Taktik; beweglicher oder stationärer Einsatz von Kräften an Verkehrswegen, um Störungen auf diesen und des dort stattfindenden Verkehrs zu verhindern

I itinéraire sécurisé - procédure de police pour s'assurer qu'un itinéraire est libre de tout danger

\begin{tabular}{cl}
108 & $\begin{array}{l}\text { situation - sum of } \\
\text { characteristic elements } \\
\text { relevant for an operation }\end{array}$ \\
\hline 109 & $\begin{array}{l}\text { (to) spread - deploy operators } \\
\text { in an area, often without } \\
\text { drawing attention }\end{array}$
\end{tabular}

108 situation - sum of characteristic elements operation in an area, often without drawing attention

\section{Lage \\ I situation initiale}

disloziert einsetzen - Einsatzkräfte abgesetzt einsetzen; außerhalb unmittelbarer Sichtweite

Idislocation - re-déploiement des forces sur une nouvelle zone et en discrétion

\begin{tabular}{lll}
\hline 110 & $\begin{array}{l}\text { squad - combination of 120 } \\
\text { operators }\end{array}$ & $\begin{array}{l}\text { Hundertschaft - ein Zusammenschluss } \\
\text { aus } 120 \text { Einsatzkräften } \\
\text { escadron }- \text { une combinaison de 120 } \\
\text { opérateurs }\end{array}$ \\
\hline 111 & $\begin{array}{l}\text { staff (headquarters) - } \\
\text { personnel qualified and } \\
\text { responsible to draft operation } \\
\text { plans, supports commanding } \\
\text { officer }\end{array}$ & $\begin{array}{l}\text { Fuipe de commandement } \\
112\end{array}$ \\
$\begin{array}{l}\text { standby police - organisation } \\
\text { within police force, with } \\
\text { specific training or technology, } \\
\text { often prepared to overcome } \\
\text { critical tasks }\end{array}$ & $\begin{array}{l}\text { Bereitschaftspolizei } \\
\text { unité en attente }\end{array}$ \\
\hline 113 & $\begin{array}{l}\text { standby unit - back-up unit } \\
\text { heiten) } \\
\text { unité en réserve }\end{array}$ \\
\hline
\end{tabular}


114 stationary - operators or technical equipment stay on their assigned location as long as their are not told otherwise, opposite to: (to) carry

\begin{tabular}{|c|c|c|}
\hline 115 & stationary traffic & $\begin{array}{l}\text { ruhender Verkehr } \\
\text { trafic stationnaire }\end{array}$ \\
\hline 116 & $\begin{array}{l}\text { task - a specific misson with } \\
\text { which a person or a group is } \\
\text { charged, leaves major } \\
\text { decisions on how to } \\
\text { accomplish the mission up to } \\
\text { head of task force, see also: } \\
\text { order }\end{array}$ & $\begin{array}{l}\text { Auftrag } \\
\text { tâche }\end{array}$ \\
\hline 117 & $\begin{array}{l}\text { task force - a temporary } \\
\text { grouping under one leader for } \\
\text { the purpose of accomplishing } \\
\text { a definite objective/a task }\end{array}$ & $\begin{array}{l}\text { Einsatzabschnitt } \\
\text { cellule de crise }\end{array}$ \\
\hline 118 & $\begin{array}{l}\text { task unit - personnel which } \\
\text { received additional training for } \\
\text { specific tasks, for example for } \\
\text { arresting trouble-makers } \\
(\mathrm{BFE})\end{array}$ & $\begin{array}{l}\text { geschlossene Einheiten mit weiterfüh- } \\
\text { renden Ausbildungen für besondere } \\
\text { Aufgaben, Beispiel: BFE } \\
\text { unité spécialisée - personnel ayant été } \\
\text { formé pour une mission spécifique, } \\
\text { exemple: BFE }\end{array}$ \\
\hline 119 & $\begin{array}{l}\text { team - a combination of } 10 \\
\text { operators }\end{array}$ & $\begin{array}{l}\text { Gruppe - ein Zusammenschluss aus } \\
10 \text { Einsatzkräften } \\
\text { groupe - une combinaison de } 10 \text { opé- } \\
\text { rateurs }\end{array}$ \\
\hline 120 & $\begin{array}{l}\text { throwing distance } \\
\text { (within/beyond) - guideline } \\
\text { for safe distances between } \\
\text { opposing groups }\end{array}$ & $\begin{array}{l}\text { Steinwurfweite (innerhalb/außerhalb) } \\
\text { dans/en dehors du champs d'action }\end{array}$ \\
\hline 121 & $\begin{array}{l}\text { unit - a small organized group } \\
\text { of personnel (military } \\
\text { expression) }\end{array}$ & $\begin{array}{l}\text { (geschlossene) Einheit } \\
\text { unité }\end{array}$ \\
\hline
\end{tabular}

vorhalten (von Einsatzkräften oder Einsatzmitteln)

I force mobile/sentinelle 
122 (to) warn of consequences by police den Hinweis geben, dass Maßnahmen erfolgen werden, wenn nicht Folge geleistet wird

I sommation

\subsection{Tactical Equipment}

123 barrier - the physical object causing a road closure

124 emergency vehicle
Absperrgerät (Beispiel: Gitter)

I barriérage

Einsatzfahrzeug

I véhicule d'urgence

125 handcuffs

Handschellen

menottes

126 personal protective equipment (PPE) tung aller Polizeibeamten)
Schutzausstattung (gehört zur Ausrüs-

I Equipments de Protection Individuelle (EPI)

127 protective helmet

Schutzhelm

casque

128 protective vest Schutzweste

IIlet par balle

129 taser - electric stun gun

Distanz-Elektro-Impulsgerät (DEIG)

I taser - pistolet à impulsion électrique (PIE)

130 truncheon - a stick-like shaped tool, part of PPE

Schlagstock

matraque

131 VAB - a specific armoured vehicle in use by French Police

VAB - ein spezifisches, gepanzertes Einsatzfahrzeug der französischen Polizei

I VAB - Véhicule de l'Avant Blindé 
Acknowledgements OPMoPS was funded by Federal Ministry of Education and Research (BMBF, grant number 13N14562) and French National Agency of Research (ANR). Benedikt Kleinmeier from OPMoPS member Munich University of Applied Science, Germany, contributed heavily to the layout.

\section{References}

[1] Bibliographisches Institut GmbH (2021): Duden, https : / /www • duden . de /

[2] dict.cc GmbH (2021): Deutsch-Englisch-Wörterbuch/Dictionnaire AllemandFrançais, https://www.dict.cc/, https://defr.dict.cc/

[3] LEO GmbH (2021): LEO, https: / / dict.leo.org/englisch-deutsch/, https://dict.leo.org/französisch-deutsch/

[4] Merriam-Webster, Inc. (2021): Merriam-Webster, https://www.merriamwebster.com/

[5] Wikimedia Foundation (2021): Wikipedia, https://de.wikipedia.org/ wiki/Wikipedia:Hauptseite, https://en.wikipedia.org/wiki/ Main_Page, https://fr.wikipedia.org/wiki/Wikip\%c3\%A9dia: Accueil_principal

[6] Arbeitsgruppe zur Erstellung eines Glossars in deutscher und französischer Sprache für die Verbesserung der grenzüberschreitenden Zusammenarbeit: Glossar deutschfranzösisch. Ministerium des Inneren und für Sport des Landes Rheinland-Pfalz (2002)

[7] Brauner, N., Hamblock, D., Schwindt, F., Spörl, U.: It's all part of the job. Englisch für die Polizei. Ein Wörterbuch. Deutsche Polizeiliteratur (2014)

[8] Bundesministerium des Inneren: Internationales Fachwortverzeichnis für Polizeibegriffe. Bundesministerium des Innern (2008)

[9] Mehlinger, H.: Fachwörtersammlung. Aus der Praxis für die Praxis. Deutschenglisch/englisch-deutsch. Gemeinsame Einsätze/Joint operations. PSW RheinlandPfalz (2012)

[10] Moreau, J.: Die Sprachen der Polizeien in Europa. 1. Wörterbuch Französisch/Deutsch. Steinbeis-Edition (2010) 